\author{
Raffaele Granata \\ (professore incaricato di Diritto canonico \\ nell’Università Popolare degli Studi di Milano)
}

\title{
La Riforma protestante \\ tra istanze di rinnovamento e movimenti ereticali *
}

The Protestant Reformation between requests for renewal and heretical movements *

ABSTRACT: The Protestant Reformation represents an epochal event as it is widely considered the true moment of transition from the Middle Ages to the modern age. Without wishing in the least to diminish the innovative significance of the aforementioned phenomenon, it is however necessary to point out how the Catholic Church, during the course of its history, has been crossed by a yearning for regeneration that has involved both the constitutional and the devotional aspects of its being. To this end, it is enough to recall the reforming work of Gregory VII, the Cluniac monastic rigor, the contribution of some regular Orders (mendicants), as well as the spread of new forms of religiosity, from the modern Devotio to the Christian humanism of Desiderio Erasmus of Rotterdam. Similarly, the originality of the Reformation did not prevent modern Protestant historiography from considering it the natural result of an evangelical pluralism already present in the twelfth century. The direct reference is to the doctrines of Pietro Valdo, John Wycliff and Jan Hus, and in particular to their ecclesiology which in various ways will be proposed by Martin Luther, with his own peculiarity, and without ever reducing himself to it.

SOMMARIO: 1. Premessa - 2. Reformatio in capite et membris - 3. La riforma gregoriana - 4. Rinnovamento monastico e nuove forme di religiosità - 5. Erasmo da Rotterdam e l'Umanesimo cristiano - 6. Ecclesia semper reformanda - 7. Gli antecedenti della Riforma - 8. I poveri di Lione - 9. Il movimento dei Lollardi - 10. La rivoluzione boema - 11 . Le guerre hussite.

\section{1 - Premessa}

Identificare le cause della Riforma protestante costituisce un'attività decisamente complessa, in quanto dobbiamo confrontarci con un fenomeno che si sviluppa in un tempo di transizione, di 'autunno del medioevo' commisto in varia misura a 'rinascenza', le cui origini sono

\footnotetext{
* Contributo sottoposto a valutazione - Article peer evaluated. Il contributo è destinato alla pubblicazione negli Studi in onore di Patrick Valdrini.
} 
tanto diverse e lontane ${ }^{1}$. Infatti questo evento, diffusamente definito epocale ${ }^{2}$, costituisce una cesura con il passato e una spinta verso un nuovo tipo di cultura, che trova la sua origine in fattori eterogenei e concomitanti ${ }^{3}$. Pertanto nessuna delle cause addotte a fondamento della 'rivoluzione protestante' spiega esaurientemente questo avvenimento ${ }^{4}$, che deve tuttavia al suo eresiarca, Martin Lutero, il proprio successo ${ }^{5}$.

${ }^{1}$ Cfr. K. BILMEYER, H. TUECHLE, L'epoca delle riforme, in Storia della Chiesa, Editrice Morcelliana, Brescia, 2008, 11ª ed., vol. III, p. 18.

2 Cfr. B. MONDIN, La Chiesa sacramento d'amore. Trattato di ecclesiologia, Edizioni Studio Domenicano, Bologna, 1993, p. 95; D. KAMPEN, Introduzione alla teologia luterana, Claudiana, Torino, 2011, p. 13.

${ }^{3}$ Come ricorda R.H. BAINTON, La Riforma protestante, Giulio Einaudi editore, Torino, 2000, p. 17, «La Riforma del secolo XVI ebbe luogo in un'età ricca di rivolgimenti tanto che quel periodo è spesso considerato il vero momento di transizione dal Medioevo all'età moderna. Parecchi movimenti vennero a svilupparsi simultaneamente. Il Rinascimento rivolse l'interesse degli uomini dal cielo alla terra, mentre le scoperte geografiche ampliarono gli orizzonti del mondo conosciuto. Il Rinascimento dimostrò maggiore entusiasmo per l'antichità classica che per quella cristiana, e contemporaneamente l'affermarsi delle monarchie nazionali indebolì il Sacro Romano Impero e minò la teocrazia papale. Intanto il sistema corporativo delle "Arti" medievali veniva disgregato dall'individualismo economico del capitalismo incipiente, che incrementava il movimento bancario e promuoveva ad un tempo l'attività affaristica. $\mathrm{E}$ in mezzo a tutto questo fermento, ecco che la Riforma venne ad infrangere la struttura monolitica di Santa Romana Chiesa».

4 Cfr. E. ISERLOH, J. GLAZIK, H. JEDIN, Riforma e Controriforma. Crisi Consolidamento - Diffusione missionaria (XVI-XVII sec.), in H. JEDIN (diretta da), Storia della Chiesa, Cooperativa Edizioni Jaca Book, Milano, 1975, vol. VI, p. 13.

${ }^{5}$ La figura di Martin Lutero è stata decisiva nel processo riformatore protestante. Infatti E. ISERLOH, J. GLAZIK, H. JEDIN, Riforma e Controriforma, cit., p. 13, osservano «che la rivoluzione avvenisse così, come oggi ci si presenta nella Riforma, è largamente dovuto a Martin Lutero, e pertanto affonda nel mistero della persona umana. Se ogni individuo è qualche cosa di ineffabile, certamente lo è Lutero, "mare di energie, di impulsi, di intuizioni e di intime esperienze"». Conformemente R. DECOT, Breve storia della Riforma protestante, Editrice Queriniana, Brescia, 2007, p. 7, sul contributo di Lutero al successo della Riforma ritiene "La trasformazione della società e della chiesa, che fu da essa provocata, si sarebbe messa in moto, in una forma o nell'altra, anche senza di lui, di fatto però fu lui il personaggio decisivo, e la sua problematica teologica influenzò in maniera determinante il corso della storia". Inoltre Y. CONGAR, Vera e falsa riforma nella Chiesa, Editoriale Jaka Book, Milano, 2010, p. 278, afferma "L'originalità di Lutero come riformatore fu di attaccare, al di là degli abusi di fatto, il sistema stesso, l'insegnamento ufficiale. È per questo, malgrado tutto, che Lutero è qualche cosa di diverso e più che un riformatore: egli è un rivoluzionario o, come si diceva una volta, in termini più conformi alla tradizione ecclesiastica, un innovatore". 


\section{2 - Reformatio in capite et membris}

La Chiesa cristiana è attraversata da un permanente anelito di rigenerazione che si è manifestato lungo il corso della sua storia, e in particolare dal XIV al XVI secolo ${ }^{6}$. Questa esigenza di rinnovamento trova la propria ragione nell'incapacità della Chiesa di corrispondere al modello introdotto da Gesù Cristo e, pertanto, nel costante tentativo di tenersi fedele alla forma evangelica7. La Chiesa ha quindi orientato la sua opera riformatrice al recupero di una forma esistente nel passato ma ormai perduta ${ }^{8}$, prestando attenzione tanto agli aspetti ecclesiologici ${ }^{9}$ quanto alla dimensione religiosa e spirituale ${ }^{10}$. Essa, in questo modo, ha sottratto ai movimenti ereticali medievali l'esclusività dell'istanza rinnovatrice ${ }^{11}$.

\section{3 - La riforma gregoriana}

${ }^{6}$ Cfr. G. PANI, Ecclesia semper reformanda dal XIV al XVI secolo, in A. SPADARO, C.M. GALLI (a cura di), La Riforma e le riforme nella Chiesa, Editrice Queriniana, Brescia, 2016, p. 127.

7 Cfr. D. SATTler, V. LePPIN (a cura di), Riforma 1517-2017. Prospettive ecumeniche, Queriniana, Brescia, 2016, p. 10.

8 Cfr. R. BATTOCCHIO, Cinquecento anni dopo. Cattolici e luterani in cammino, Messaggero di Sant'Antonio, Padova, 2017, p. 29.

9 Infatti Y. CONGAR, Vera e falsa, cit., pp. 271-272, ricorda che «Nel latino profano, le parole reformare, reformatio significavano: dare a un oggetto un'altra forma, riportarlo alla sua forma primitiva; quindi: correggere dei costumi corrotti. [...] A partire dal secolo XI tuttavia, compare un uso nuovo, o almeno una nuova applicazione dell'uso antico: si applica il termine reformatio a delle realtà sociali come tali. E in primo luogo alla Chiesa, alle istituzioni e agli uomini di Chiesa, che si tratta di ricondurre, non solamente all'osservanza dei santi canoni, ma all' „ecclesiae primitivae forma" ».

10 Ad avviso di R. BATTOCCHIO, Cinquecento anni dopo, cit., p. 28, «chi esorta alla riforma parte da una constatazione: per qualche motivo, una realtà è stata de-formata, ha perso la propria forma, non è come dovrebbe e potrebbe essere. Il termine "forma", in questo contesto non si riferisce all'aspetto esteriore delle cose, ma allude alla verità della cosa stessa, a ciò che ne definisce l'identità e che permette di riconoscerla. C'è una forma del discepolo (del cristiano, della cristiana), c'è una forma della chiesa: l'una e l'altra sono determinate dal riferimento a Gesù Cristo, alla sua parola e alla sua vita».

11 Ciò è testimoniato, innanzitutto, dal diffuso utilizzo in ambito cattolico del termine reformatio, che ha assunto il significato di ritorno a uno stato originario. A tal proposito, D. SATTLER, V. LEPPIN (a cura di), Riforma, cit., p. 46, ricordano che l'impiego di tale espressione "compare già nel tardo Medioevo con riferimento a programmi volti a un rinnovamento fondamentale della chiesa (ripristino di quanto era stato deformato)". 
Fondamentale nel processo di rinascenza ecclesiastica, che coinvolse la Chiesa d'Occidente a partire dal secolo XI'2 , è il contributo di Gregorio VII, il quale intese sottrarre il Papato al controllo imperiale e assicurare un rinnovamento morale e costituzionale delle strutture ecclesiastiche ${ }^{13}$.

Per conseguire tale risultato, la riforma gregoriana ripropone innanzitutto il concetto di primato pontificio da intendersi "come potere unico, universale, che non ha eguali nella Chiesa e nel mondo, e può assoggettare a sé ogni altra istituzione ecclesiastica o politica"14. I contenuti di questa primazia sono formulati analiticamente nei dictatus papae $^{15}$, in cui si afferma che il Pontefice domina sovrano sulla Chiesa poiché «tale potere si fonda sul privilegio divino di rappresentare il successore di Pietro (vicarius Petri), a cui è stata conferita la pienezza dei poteri mediante la "successione" nella Sede apostolica di Roma» ${ }^{16}$.

Nel citato documento, viene altresì teorizzata, in modo inequivocabile, la supremazia del Pontefice sull'Impero e sugli Imperatori ${ }^{17}$, per la cui attuazione risulta strumentale $1^{\prime}$ aver riservato l'elezione del Papa al Conclave ${ }^{18}$ e l'aver ingaggiato la lotta delle investiture per liberare le nomine ecclesiastiche da interferenze temporali, considerate portatrici di grande corruzione, conclusasi con il Concordato

${ }^{12}$ Cfr. C. CARDIA, Principi di diritto ecclesiastico. Tradizione europea legislazione italiana, Giappichelli, Torino, 2010, 3a ed., p. 35.

${ }^{13}$ Cfr. C. FANTAPPIÈ, Storia del diritto canonico e delle istituzioni della Chiesa, il Mulino, Bologna, 2011, p. 89.

${ }^{14}$ Cfr. C. CARDIA, Principi di diritto ecclesiastico, cit., p. 37. Inoltre ricorda L. FELICI, La Riforma protestante nell'Europa del Cinquecento, Carocci, Roma, 2016, p. 17, come il progetto teocratico della Chiesa ovvero "la supremazia e autorità assoluta del pontefice nella sfera temporale e spirituale fu proclamata da Gregorio VII nel 1075 nella lotta per le investiture e ribadita nei due secoli successivi da Innocenzo III e da Bonifacio VIII".

15 I dictatus papae sono appunti di Gregorio VII, probabilmente non destinati alla pubblicazione, la cui natura appare tutt'oggi controversa. Si veda sul punto G.M. CANTARELLA, Il sole e la luna. La rivoluzione di Gregorio VII papa 1073-1085, Laterza, Roma-Bari, 2005.

${ }^{16}$ Cfr. C. FANTAPPIÈ, Storia del diritto canonico, cit., p. 96.

${ }^{17}$ Cfr. C. CARDIA, Principi di diritto ecclesiastico, cit., p. 38.

18 Per J. ORLANDIS, Le istituzioni della Chiesa cattolica. Storia, diritto, attualità, San Paolo, Cinisello Balsamo, 2005, p. 37, "il primo obiettivo dell'azione dei riformatori gregoriani fu quello di stabilire un procedimento specifico per l'elezione pontificia, diverso da quello tradizionale in uso per le nomine vescovili. L'elezione papale doveva essere affidata esclusivamente a un ridotto collegio di chierici, i cardinali". 
di Worms del 1122 tra Enrico V e Callisto II ${ }^{19}$.

La riforma gregoriana cercò inoltre di porre rimedio alla crisi dei costumi del basso clero, che era solito sposarsi o convivere, provocando scandalo nella comunità dei fedeli. Per porre fine alla pratica del concubinato, Gregorio VII rinnovò il divieto di matrimonio per i sacerdoti, già fissato nel Sinodo del Laterano del 105920, che divenne legge generale della Chiesa d'Occidente con il I Lateranense del $1123^{21}$. Le ragioni poste a fondamento del celibato ecclesiastico sono molteplici:

"molto forte è la motivazione ascetico - morale che vede nella continenza e nella castità gli strumenti privilegiati per il perfezionamento spirituale dell'individuo". Decisiva è "la motivazione pastorale - istituzionale, per la quale il matrimonio impedirebbe al clero di costituirsi in personale ecclesiastico stabile e autonomo rispetto agli impegni mondani".

Non deve essere sottovalutata

"la motivazione economico-proprietaria della regola celibataria. Un prete sposato inevitabilmente deve far fronte alle esigenze economiche della famiglia, la quale altrettanto inevitabilmente porrebbe dei problemi ereditari anche nei confronti della proprietà ecclesiastica affidata al singolo sacerdote" 22 .

Infine Gregorio VII licenziò una vera e propria rifondazione costituzionale della Chiesa, passata alla storia come 'centralizzazione gregoriana'. Essa, sul modello della Congregazione cluniacense, si distinse per la previsione di un concetto di Chiesa come organismo gerarchico dipendente dalla Chiesa romana, al vertice del quale sta il Papato ${ }^{23}$, che si dotò della collaborazione di un complesso di istituzioni destinate a rimanere per lungo tempo nell'amministrazione centrale della Chiesa: il Concistoro, gli Uffici (la Cancelleria, la Camera apostolica e la Dataria) e i

${ }^{19}$ Come ricorda C. CARDIA, Principi di diritto ecclesiastico, cit., p. 37, "L'accordo non segna una vittoria completa del papato, si limita a distinguere tra l'investitura canonica e i regalia appartenenti alla Chiesa romana, e l'investitura temporale che spetta all'imperatore".

${ }^{20}$ Cfr. C. FANTAPPIÈ, Storia del diritto canonico, cit., p. 94.

${ }^{21}$ Il Laterano I inaugura una serie di sette Concili, conclusa a Vienne nel 1311-1312, passati alla storia come Concili papali in quanto, come afferma N.P. TANNER, I Concili della Chiesa, Jaka Book, Milano, 1999, p. 61, "la convocazione, la presidenza e la promulgazione dei decreti era tutta opera del papa in persona o attraverso delegati".

${ }^{22}$ Cfr. C. CARDIA, Principi di diritto ecclesiastico, cit., p. 36.

${ }^{23}$ Cfr. C. FANTAPPIÈ, Storia del diritto canonico, cit., p. 94. 
Tribunali (la Audientia Sacri Palatii o Tribunale della Rota, la Segnatura apostolica e la Penitenzieria) ${ }^{24}$.

Nell'elaborazione del progetto di riforma brevemente descritto ha influito, senza dubbio, l'esperienza personale di Ildebrando di Soana che, prima di essere eletto al soglio pontificio con il nome di Gregorio VII, aveva vissuto la condizione di monaco cluniacense. Infatti, già nel $\mathrm{X}$ secolo si erano manifestati segni di rinnovamento all'interno della Chiesa, orientati a restituirle il ruolo di guida spirituale della cristianità, di cui il Monastero di Cluny, posto nella Francia meridionale, fu un fulgido esempio.

Il movimento di riforma cluniacense riguardò innanzitutto la vita monastica che, dimidiando la regola di Benedetto dell'ora et labora, fu orientata esclusivamente alla preghiera e alla meditazione delle Sacre Scritture ${ }^{25}$, lasciando ai laici la responsabilità del lavoro per le esigenze dell'Abbazia $^{26}$. Inoltre, sotto il profilo ecclesiastico, Cluny rappresentò un Monastero 'esente', cioè "libero da qualsivoglia potere di signoria laica o di autorità episcopale, e sottomesso direttamente al Papa"27.

\section{4 - Rinnovamento monastico e nuove forme di religiosità}

Lo spirito di rinnovamento monastico venne ereditato, nel XIII secolo, da

${ }^{24}$ Cfr. J. ORLANDIS, Le istituzioni, cit., pp. 46-54. La descrizione e il funzionamento degli organismi e delle istituzioni ecclesiastiche de quibus è contenuta in P.G. WALLACE, La lunga età della Riforma, il Mulino, Bologna, 2006, pp. 56-57.

25 Ricorda C. CARDIA, Principi di diritto ecclesiastico, cit., p. 41, che "i monaci cluniacensi si specializzarono [...] nella preghiera a favore delle anime dei defunti (sono $i$ tempi in cui si afferma l'idea di purgatorio), stipulando dei contratti con case regnanti europee, con principi e nobili di ogni livello, nei quali figura l'obbligo dei religiosi di fare preghiera (e liturgia) a favore dei familiari scomparsi, in cambio di prestazioni in denaro o in altra forma (fondi, privilegi, concessioni)".

26 Sul punto C. CARDIA, La Chiesa tra storia e diritto, Giappichelli, Torino, 2010, p. 270, precisa che "al lavoro provvedono i servi della gleba che si sistemano con le proprie famiglie attorno a Cluny e alle sue succursali prefigurando futuri stanziamenti cittadini".

27 Cfr. J. ORLANDIS, Le istituzioni, cit., p. 161. Sulla natura giuridica del monastero esente, AA. VV., Ugo abate di Cluny. Splendore e crisi della cultura monastica, a cura di G. CANTARELlA, D. TUNIZ, 2a ed., Europìa, Novara, 1991, pp. 10-11, precisano che «l'abbazia borgognona di Cluny venne fondata nel 910 da Guglielmo d'Aquitania sui beni familiari (allodiali) [...] e non in terre appartenenti al fiscus regio [...] e dal momento [...] che era stata fondata su terre private e non su terre del fisco, essa si trovò [...] svincolata da qualunque autorità o forma di controllo delle autorità secolari: era lo stato di "immunità"». 
alcuni Ordini regolari che decisero, diversamente dai vecchi Monasteri sorti in luoghi appartati, di insediarsi con i propri Conventi nelle città, divenute medio tempore base del sistema urbano europeo ${ }^{28}$. Questi frati, chiamati 'mendicanti', perché sostenuti dalle elemosine dei fedeli, si fecero promotori di un nuovo modello di vita religiosa nella quale, diversamente dal monachesimo benedettino, non ci si dedicava esclusivamente alla vita di preghiera e di studio, ma alla predicazione e all'assistenza dei poveri e dei malati ${ }^{29}$.

Gli Ordini mendicanti più importanti furono i francescani e i domenicani ${ }^{30}$, fondati rispettivamente da Francesco d'Assisi e Domenico di Guzmán, che Dante Alighieri considera inviati dalla Provvidenza per risollevare le sorti della cristianità ${ }^{31}$. In particolare $i$ francescani praticarono un modello di assoluta povertà ${ }^{2}$, individuale e collettiva, impegnandosi nell'apostolato missionario e distinguendosi per un'elevata spiritualità, mentre i domenicani si dedicarono maggiormente alla predicazione, alla difesa della fede contro le eresie e all'insegnamento nelle Università dell'Occidente cristiano ${ }^{33}$.

Sul modello domenicano si costituì, inoltre, l'Ordine degli eremitani agostiniani, impegnati nelle Università e nella predicazione cittadina, tra i quali si distinse la figura di Martin Lutero, che il 17 luglio 1505 entrò, come novizio, nel Convento di Erfurt ${ }^{34}$ di cui era Vicario Johann von Staupitz ${ }^{35}$. A costui Lutero dovrà gran parte della sua fortuna accademica, ricoprendo, su sua chiamata, la cattedra di Etica e Sacra Scrittura

${ }^{28}$ Cfr. J. ORLANDIS, Le istituzioni, cit., p. 164.

${ }^{29}$ Cfr. P.G. WALLACE, La lunga età, cit., p. 55.

${ }^{30}$ Cfr. C. CARDIA, La Chiesa tra storia e diritto, cit., pp. 277-282.

31 Cfr. D. ALIGHIERI, La Divina Commedia, Paradiso, XI, 28-39, per il quale "La provedenza, che governa il mondo con quel consiglio nel quale ogni aspetto creato è vinto pria che vada al fondo, però ch'andasse ver lo suo diletto la sposa di colui ch'ad alte grida, disposò lei col sangue benedetto, in sé sicura e anche a lui più fida, due principi ordinò in suo favore che quinci e quindi le fosser per guida. L'un fu tutto serafico in ardore; l'altro per sapienza in terra fue di cherubica luce uno splendore".

32 L'interpretazione, più o meno rigorosa, dei precetti riguardanti la povertà determinò dapprima la scissione tra la parte maggioritaria dell'Ordine e gli spirituali, confluiti nella setta eretica dei fraticelli (XIII secolo), e successivamente la divisione tra gli osservanti e i conventuali (XVI secolo). Cfr. J. ORLANDIS, Le istituzioni, cit., p. 165.

${ }^{33}$ Cfr. J. ORLANDIS, Le istituzioni, cit., p. 166.

${ }^{34}$ Cfr. G. DALL'OLIO, Martin Lutero, Carocci, Roma, 2013, p. 44.

${ }^{35}$ Cfr. G. EBELING, Lutero: l'itinerario e il messaggio, Claudiana, Torino, 1998, pp. 3031. 
all'Università di Wittenberg, istituita qualche anno prima dal Duca di Sassonia, Ludovico il Savio ${ }^{36}$.

L'opera di rinnovamento realizzata dalla Chiesa in questi secoli non riuscì però ad arrestare il suo processo di decadenza che si tradusse, quantomeno nel giudizio della storiografia classica, nella principale causa della Riforma protestante ${ }^{37}$. Nonostante tutto, la cristianità continuò a esprimere nuove forme di devozione e ad elaborare modelli di riorganizzazione ecclesiastica annunciati e mai attuati. In particolare l'azione pastorale degli Ordini mendicanti rappresentò, soprattutto in Germania, il volano per la diffusione di una nuova mistica che esercitò una forte attrattiva sul clero, sui religiosi e perfino sui laici. I seguaci di questo movimento, chiamati 'amici di Dio', si opposero al rilassamento morale del tempo, promuovendo un rinnovato senso religioso e culturale, che aspirava a una totale libertà dalle passioni e dall'attaccamento al mondo, per giungere all'Assoluto.

Ragioni politiche favorirono la diffusione del fenomeno anche nei Paesi Bassi, dove si sviluppò una nuova forma di ascetismo con l'associazione dei 'Fratelli e sorelle della vita comune' 38 , nata dall'iniziativa di Geert de Groote, che nel 1401 ottenne l'approvazione ufficiale del Vescovo di Utrecht. In questa comunità laicale si sviluppò una forma di pietà nota come Devotio moderna ${ }^{39}$ che, guardandosi bene dal richiedere un cambiamento delle principali strutture della Chiesa ${ }^{40}$, "poneva l'accento principale sull'imitazione di Cristo e sull'esercizio di una mistica piena di misura"41. Infatti la traccia più evidente di questa religiosità è il $D e$ imitazione Christi di Tommaso da Kempis, che rappresenta uno dei libri

${ }^{36}$ Cfr. R. COGGI, La Riforma Protestante. Martin Lutero, Studio Domenicano, Bologna, 2004, vol. II, p. 9.

37 Cfr. B. MONDIN, La Chiesa sacramento d'amore, cit., pp. 95-96.

38 Cfr. S. PEYRONEL RAMBALDI, La Riforma protestante, Claudiana, Torino, 2017, p. 4 .

39 Secondo G. TOURN, I Protestanti, una rivoluzione. 1. Dalle origini a Calvino (15171564), Claudiana, Torino, 1993, p. 21, questa "corrente spirituale [...] si definisce abitualmente come la Devotio moderna, la devozione, la pietà moderna, per contrapporla a quella antica praticata nell'età medievale fino al XIV secolo e che aveva come centro i monasteri. Nella Devotio moderna la fede è più convinzione e sentimento personale che pratica religiosa, è centrata sull'individuo, le sue attese, la sua sensibilità".

${ }^{40}$ Cfr. W.G. NAPHY, La rivoluzione protestante. L'altro Cristianesimo, Cortina, Milano, 2010, p. 8.

${ }^{41}$ Cfr. K. BILMEYER, H. TUECHLE, L'epoca delle riforme, cit., p. 138. 
prediletti della cristianità 42 .

Inoltre l'impegno laicale a condurre una vita devota portò allo sviluppo di una meditazione regolare e metodica e, quindi, a una vera riflessione teologica, nota come 'teologia della pietà', secondo la quale la salvezza si raggiunge non attraverso i sacramenti e le opere buone bensì attraverso l'incontro personale, intimo con $\mathrm{Dio}^{43}$.

\section{5 - Erasmo da Rotterdam e l'Umanesimo cristiano}

Nella medesima direzione della Devotio moderna, ma con caratteristiche e diffusione differenti, si colloca l'Umanesimo ${ }^{44}$. Questa corrente, sorta in Italia durante il Rinascimento, si propone di recuperare la cultura antica favorendo le lingue classiche e, in ambito religioso, l'ebraico ${ }^{45}$. Tuttavia la forma di Umanesimo più aderente al tema del 'rinnovamento' è quella che si sviluppa nell'Europa settentrionale ${ }^{46}$, e specificamente in Germania, laddove gli umanisti, in prevalenza chierici, riprendono lo studio delle Sacre Scritture, riproponendone il significato originale ${ }^{47}$.

Il massimo esponente di questo "umanesimo cristiano" 48 , a tratti

${ }^{42}$ Cfr. G. TOURN, I Protestanti, cit., p. 21.

${ }^{43}$ Cfr. D. SATTLER, V. LEPPIN, Riforma, cit., pp. 53-54.

${ }_{44}$ L'Umanesimo è anche definito l'aspetto letterario del Rinascimento. Cfr. G. MARTINA, Storia della Chiesa. Da Lutero ai giorni nostri. L'età della Riforma, Morcelliana, Brescia, 1993, vol. I, p. 89. Specificano ulteriormente K. BILMEYER, H. TUECHLE, L'epoca delle riforme, cit., p. 158, che questa corrente «da principio [...] si presenta generalmente come una "rinascita" dell'antichità classica; per quanto essa riguarda il campo degli studi, dell'educazione umana, delle scienze e delle lettere si preferisce indicarla col nome di umanesimo; invece per i riflessi che ne derivano nell'arte, nella civiltà, nella politica, nella visione del mondo e della vita, si denomina piuttosto rinascimento». Per un approfondimento tematico si rinvia, tra gli altri, a G. TOFFANIN, Storia dell'Umanesimo. L'Umanesimo italiano. (Dal XIV al XVI secolo), Zanichelli, Bologna, 1964, vol. II.

${ }^{45}$ Cfr. W.G. NAPHY, La rivoluzione protestante, cit., p. 10.

46 Sul punto diffusamente G. TOFFANIN, Storia dell'Umanesimo. La fine del logos. (L'Umanesimo europeo), Zanichelli, Bologna, 1964, vol. III.

${ }^{47}$ Cfr. P.G. WALLACE, La lunga età, cit., p. 98.

48 Le posizioni dell'umanesimo cristiano sono sinteticamente descritte da É.G. LÉONARD, La Riforma: dalle origini al 1564, in Storia del Protestantesimo, il Saggiatore, Milano, 1971, vol. I, pp. 35-42. 
austero e rigoroso, fu Desiderio Erasmo da Rotterdam ${ }^{49}$, il cui pensiero "costituì la sintesi più compiuta delle aspirazioni di riforma religiosa e intellettuale allora urgenti, e una risposta affatto innovativa ad esse" Egli, infatti, fu dapprima educato alla religiosità della Devotio moderna a Deventer ${ }^{51}$, e successivamente entrò nel Convento agostiniano di Stein, dove lesse Lorenzo Valla e si allontanò dal monachesimo ${ }^{52}$. Attraverso la sua copiosa letteratura, dall'Enchiridion militis christiani (1502) al Novum Instrumentum omne (1516), passando per l'Enkomion Moris (1509),

"l'umanista formò generazioni di giovani ad un sapere mirante al miglioramento individuale e dall'intera Societas Christiana, sulla base dei principi della pace, della libertà, della tolleranza, della ragione critica, di una nuova religiosità spirituale e della cultura classica" 53 .

Erasmo non si sottrasse dal rivolgere critiche alle ingiustizie del suo tempo e al malcostume della Chiesa che gli valse, in un primo momento, anche la vicinanza di Lutero. È noto, infatti, come solo nel 1524 i due entrarono pubblicamente in conflitto sulla questione dell'arbitrio umano: "secondo Erasmo l'uomo era libero di scegliere se seguire la parola di Dio oppure no, mentre Lutero considerava l'uomo libero solo se sottomesso al volere divino" 54 .

In questo modo veniva sancita la rottura tra l'Umanesimo e la Riforma, anche se tuttora sussistono incertezze sulle effettive ragioni che la determinarono. Secondo alcuni, esse sono legate a questioni personali o di temperamento ${ }^{55}$, per altri, alla volontà di Lutero di non attribuire più "alla natura umana la capacità di scegliere tra il bene e il male, tornando almeno così sembra - al medievale primato del divino sull'umano che il Rinascimento era stato sul punto di scalzare" 56.

Indipendentemente dalla fedele ricostruzione della vicenda dianzi 1988.

49 Cfr. C. AUGUSTIJN, Erasmo da Rotterdam. La vita e l'opera, Morcelliana, Brescia,

${ }^{50}$ Cfr. L. FELICI, La Riforma protestante, cit., p. 37.

51 Cfr. S. PEYRONEL RAMBALDI, La Riforma protestante, cit., p. 4.

52 Cfr. É.G. LÉONARD, La Riforma, cit., p. 40. Inoltre, sottolinea la sua posizione antiscolastica J.P. TORREL, La teologia cattolica, Jaca Book, Milano, 1998, p. 33, per il quale "la decadenza della teologia scolastica provocò varie reazioni. La più nota è quella degli umanisti, di cui Erasmo (1469-1536) è il rappresentante principale".

53 Cfr. J.P. TORREL, La teologia cattolica, cit., p. 33.

${ }^{54}$ Cfr. P.G. WALLACE, La lunga età, cit., pp. 99-100.

55 Cfr. G. PANI, Ecclesia semper reformanda, cit., p. 132.

${ }^{56}$ Cfr. G. DALL'OLIO, Martin Lutero, cit., p. 121. 
descritta, appare innegabile come la stessa sia stata favorita dalle posizioni, meno radicali, che Erasmo assunse nei confronti della riforma della Chiesa, da intendersi ad intra, conformemente all'ideale di unità religiosa per lui prioritaria ${ }^{57}$.

\section{6 - Ecclesia semper reformanda}

In modo del tutto speculare, e negli stessi secoli ai quali ci siamo finora riferiti, la Chiesa, consapevole delle proprie miserevoli condizioni, ha avanzato numerose proposte di riforma, con lo scopo di rinnovarsi 'nel capo e nelle membra'. Significativo è, a tal proposito, quanto accaduto durante la celebrazione del Concilio di Vienne (1311-1312), nel quale Papa Clemente $\mathrm{V}$ invitò i Vescovi presenti a formulare proposte di riforma della Chiesa universale. Tra queste merita menzione il trattato ${ }^{58}$ del Vescovo di Mende, Guillaume Durand, che coniò l'espressione reformatio tam in capite quam in membris, ripresa anche nei Concili ecumenici successivi (in particolare Costanza 1414-1418 e Basilea 1431-1449) ma scarsamente attuata $^{59}$.

Infatti,

"per due secoli, non si fece nessun tentativo veramente serio per attuare una tale riforma. È venuta a crearsi allora, e ad affermarsi con sempre maggior vigore, la convinzione che, al di là degli abusi particolari, occorreva riformare il sistema stesso; che se la testa non era disponibile, il corpo doveva portare avanti questa riforma senza di essa, e occorrendo contro di essa" 60 .

57 Cfr. L. FELICI, La Riforma protestante, cit., p. 37. Più incerto è G. ALBERIGO, La Riforma protestante, origini e cause, 2a ed., Queriniana, Brescia, 1988, p. 21, per il quale "si è potuto e si potrà all'infinito vedere in Erasmo, e nell'umanesimo pio, ora dei precursori e degli anticipatori di motivi protestanti, ora degli esponenti di riforma cattolica che esistevano all'interno della chiesa. In una parola, non si può dare un'etichetta definita a questo movimento degli spiriti". Infatti per E. ISERLOH, J. GLAZIK, H. JEDIN, Riforma e Controriforma, cit., p. 172, "se l'umanesimo, con i suoi studi sul testo biblico e con la sua critica alla chiesa, aveva molto in comune con la Riforma sotto l'aspetto formale, esso però - con il suo ottimismo etico, e anche con il suo moralismo - si distaccava dal principio materiale della sola fides e della sola gratia, più di quanto la maggioranza dei contemporanei ne fosse consapevole".

58 Cfr. G. DURANTIS, Tractatus de modo generalis concilii celebrandi et corruptelis in ecclesia reformandis, Lyon, 1531.

${ }^{59}$ Cfr. G. PANI, Ecclesia semper reformanda, cit., p. 128.

${ }^{60}$ Cfr. Y. CONGAR, Vera e falsa, cit., p. 287. 
Questo appello trovò puntuale accoglimento in Germania che, durante la seconda metà del XV secolo, viveva una condizione particolarmente grave: infatti insostenibile era divenuta, per il clero teutonico, la pressione della Curia pontificia che, manifestandosi in un elevato fiscalismo, aveva sollecitato un forte sentimento antiromano ${ }^{61}$. Ciò portò la Dieta di Francoforte del 1456, sotto la presidenza dell'Arcivescovo Teodorico di Magonza, a formulare i Gravamina nationis Germanicae, un complesso di lagnanze nelle quali "si reclamava contro l'estorsione di danaro da parte della Curia romana sotto vari pretesti: tasse, contributi, casi riservati, indulgenze" 62 . Qualche anno più tardi, sarà Martin Lutero a raccogliere queste lamentele, condensandole nel primo dei suoi tre grandi scritti riformatori del 1520, dal titolo "Alla nobiltà cristiana della nazione tedesca"63.

È quindi paradossale che il Concilio Lateranense V (1512-1517) venga presentato come l'estremo tentativo di riforma della Chiesa in capite et membris, e che Papa Leone X, nel 1514, conceda ad Alberto di Brandeburgo, già Arcivescovo di Magdeburgo e Amministratore di Halberstadt, il Vescovado di Magonza dietro il pagamento di un'ingente somma di danaro, da finanziare mediante la vendita di indulgenze bandita sui suoi territori64. $\mathrm{Fu}$, infatti, questo accadimento a determinare l'affissione, sulla porta della Chiesa del Castello di Wittemberg, delle novantacinque tesi luterane ${ }^{65}$, oggetto di una recente rilettura storiografica, e la conseguente riflessione teologica ${ }^{66}$.

${ }^{61}$ Cfr. G. MARTINA, Storia della Chiesa, cit., p. 115.

${ }^{62}$ Cfr. B. MONDIN, Storia della Teologia, Studio Domenicano, Bologna, 1996, vol. III, p. 135. Inoltre, secondo K. BILMEYER, H. TUECHLE, L'epoca delle riforme, cit., p. 201, "l'imperatore Massimiliano nel 1510 incaricò un noto umanista Giacomo Wimpfeling di farne una nuova compilazione e qualche anno dopo, nelle diete di Augusta del 1518, di Worms del 1521 e di Norimberga nel 1522-23, i Gravamina col loro numero elevato (100, oppure 102) e la loro aspra formulazione costituirono, pur rimanendo solo allo stato di progetto, un'arma efficace di agitazione per i novatori delle cose di religione".

${ }^{63}$ Cfr. M. LUTHER, An den christlichen Adel deutscher Nation von des christlichen Standes Besserung (1520), WA 6, 381-469, traduzione italiana di G. PANZIERI SAIJAM, Alla nobiltà cristiana di nazione tedesca, in MARTIN LUTERO, Scritti politici, UTET, Torino, 1978, p. 123 ss.

${ }^{64}$ Cfr. G. ALBERIGO, La Chiesa e l'Europa nel Cinquecento, in La storia dei giubilei, II: 1450-1575, Giunti, Firenze, 1998, pp. 150-179.

${ }^{65}$ Sul tema si rinvia a P. RICCA, G. TOURN, Le 95 tesi di Lutero, Claudiana, Torino, 2016.

${ }^{66}$ Cfr. L. MAGGI, A. REGINATO, La Riforma protestante tra passato e presente, Studio Domenicano, Bologna, 2004, p. 27. 
Bisogna attendere il Concilio di Trento67 (1545-1563) e, in special modo, la sua terza sessione, affinché la Chiesa di Roma passi al contrattacco $^{68}$, vincendo definitivamente l'immobilismo pontificio, frutto della paura del Conciliarismo ${ }^{69}$. Questa nuova fase fu preceduta dall'attività del Consilium de emendanda ecclesia, istituito da Paolo III nel 1537, che propose "un progetto di riforma della Chiesa di carattere eminentemente pastorale", avendo "come scopo la cura animarum" sul presupposto della "constatazione della vita mondana e scandalosa della Chiesa, che ha origine innanzitutto dal Papato"70.

Indipendentemente dai risultati raggiunti, l'organismo in parola ebbe il merito di aver aperto la strada alla "controriforma"71.

\section{7 - Gli antecedenti della Riforma}

Lo spirito riformatore di Martin Lutero, manifestatosi nella Sassonia del XVI secolo, rappresenta un unicum nel panorama spirituale ed ecclesiologico del tempo, eguagliato, per successo e diffusione, solo da Giovanni Calvino ${ }^{72}$.

\footnotetext{
${ }^{67}$ Per un approfondimento tematico si rinvia a H. JEDIN, Storia del Concilio di Trento, Morcelliana, Brescia, 2010.

${ }^{68}$ Cfr. G.R. ELTON, Storia del Mondo Moderno. La Riforma (1520-1559), Aldo Garzanti, Milano, 1967, vol. II, p. 3.

${ }^{69}$ Cfr. R. DECOT, Breve storia, cit., p. 139. Conformemente N.P. TANNER, I Concili della Chiesa, cit., p. 87, ricorda che «quando Martin Lutero venne in conflitto con l'autorità ecclesiastica sulle indulgenze, si appellò in primo luogo al concilio generale, promettendo di sottomettersi alla sua decisione se fosse stato convocato; anche se ben presto egli si appellò alla Bibbia. Fu, tuttavia, quasi trent'anni dopo la sua iniziale rottura con la chiesa romana nel 1517 che si riunì il concilio di Trento, nel 1545, lasso di tempo in cui la Riforma si era diffusa ampiamente e le ferite all'interno della chiesa si dimostrarono troppo profonde per essere sanate. Ci furono diverse ragioni per questa lunga dilazione. Un'importante ragione fu il timore di un ritorno del "fantasma conciliare"».

${ }^{70}$ Cfr. G. PANI, Ecclesia semper reformanda, cit., p. 139.

${ }^{71}$ Cfr. D. SATTLER, V. LEPPIN, Riforma, cit., p. 48, secondo i quali «il termine fu introdotto per descrivere il comportamento della chiesa antica in relazione alla Riforma, integrando quel concetto con quello di "riforma cattolica", il che rende evidente che anche da parte cattolica furono raccolti ed attuati impulsi di riforma della chiesa del tardo Medioevo».

72 Sulla figura di Giovanni Calvino e sul contesto territoriale della sua azione riformatrice si vedano, rispettivamente, A.E. MCGRATH, Giovanni Calvino. Il Riformatore e la sua influenza sulla cultura occidentale, 3a ed., Claudiana, Torino, 2009, e S. RONCHI, Zwingli e Calvino nel contesto elvetico, Studio Domenicano, Bologna, 2005, pp. 87-122.
} 
L'originalità della Riforma non impedisce, comunque, di considerarla l'approdo naturale di un pluralismo evangelico già presente nel XII secolo ${ }^{73}$, che pretendeva un cambiamento della Chiesa eliminando "ciò che si era aggiunto nei secoli", e riportando "le cose a come Dio le aveva concepite"74. Infatti mentre "le azioni di riforma mirano a rinnovare le forme di vita della Chiesa" introducendo, come avvenuto in ambito cattolico, innovazioni "nell'organizzazione, nella pratica e nella struttura", "la Riforma mira a dare forma agli attributi essenziali e tende quindi al rinnovamento della Chiesa"75.

Questo concetto, sintetizzato nella formula Ecclesia semper reformand $a^{76}$, esprime un processo volto a ripristinare quanto nella Chiesa era stato deformato dalla compromissione della gerarchia ecclesiastica ${ }^{77}$, come anticipato, a più riprese, dai movimenti eretici popolari ${ }^{78}$. A tal proposito, trova particolare seguito, in ambito protestante, l'idea che la Riforma rappresenti lo sbocco di una catena di eresie ${ }^{79}$, delle quali recepisce alcuni principi cardine, pur senza ridursi a essi, opportunamente condensati nelle dottrine di Pietro Valdo, John Wycliff e Jan Hus ${ }^{80}$.

${ }^{73}$ Cfr. P. DE LIGNEROLLES, J.P. MEYNARD, Storia della spiritualità cristiana, Piero Gribaudi, Milano, 2005, p. 165.

${ }^{74}$ Cfr. M. RUBBOLI, I protestanti, il Mulino, Bologna, 2007, p. 8.

${ }^{75}$ Cfr. D. SATTLER, V. LEPPIN, Riforma, cit., p. 85.

${ }^{76}$ L'espressione, letteralmente 'la Chiesa deve sempre essere riformata', trova una particolare diffusione in ambito calvinista, anche se incerta ne è l'origine. A tale proposito, G. PANI, Ecclesia semper reformanda, cit., pp. 126-127, sottolinea che la formula Ecclesia semper reformanda è meglio documentata nella "voce Reformation di Theodor Mahlmann, nell'Historisches Wörterbuch der Philosophie del 1992 [...] che ne riconduce l'origine all'olandese Jocodus van Lodensteyns (1602-1677), pastore riformato della corrente pietista più rigorosa e impegnata, il quale a sua volta rinvia ad una fonte anonima".

77 Cfr. P. GAJESWKI, La Riforma in Francia, nei Paesi Bassi, in Scandinavia e in Europa orientale, Studio Domenicano, Bologna, 2007, p. 9.

${ }^{78}$ Cfr. K. BILMEYER, H. TUECHLE, L'epoca delle riforme, cit., p. 24.

${ }^{79}$ Questa ricostruzione è contestata da É.G. LÉONARD, La Riforma, cit., p. 27, per il quale le eresie "al contrario erano in profondo declino, e talvolta esaurite, e quindi da esse sicuramente non potè sorgere un movimento esteso e potente come la rivoluzione religiosa del XVI secolo".

${ }^{80}$ Infatti secondo G. MARTINA, Storia della Chiesa, cit., p. 109, "non si può [...] sottovalutare il fatto che quasi tutte le tesi difese più tardi da Lutero, almeno nell'ecclesiologia, erano state già esposte [...] soprattutto da questi tre autori". In questa direzione Y. CONGAR, Vera e falsa, cit., pp. 281-283, sottolinea che l'ecclesiologia di Wycliff e Hus sia espressione di un certo agostinismo nel quale si realizza "una disgiunzione, se non una opposizione, tra la Comunione con Cristo, interamente spirituale e pura, e l'inserimento nella Chiesa visibile". Per questi autori, nei quali la 


\section{8 - I poveri di Lione}

Pietro Valdo è un mercante vissuto a Lione negli anni 1170-1180, che decide di abbandonare la propria ricchezza, donandola ai poveri, e di predicare la penitenza mediante la lettura dell'Evangelo, alla stregua dei primi discepoli di Cristo ${ }^{81}$. I suoi seguaci, chiamati in modo dispregiativo valdesi, si definiscono 'i poveri di Lione', o più precisamente 'poveri in Spirito' per testimoniare la fedeltà alla povertà evangelica e il legame di questa comunità con il discorso della Montagna ${ }^{82}$, in particolare con la prima Beatitudine ${ }^{83}$, considerato il testo più importante per la vita della fede ${ }^{84}$.

L'esperienza personale e confessionale di Valdo rimanda, inevitabilmente, alla vicenda umana e religiosa di Francesco d'Assisi che del primo condividerà, in una generazione posteriore, la classe sociale di appartenenza e la necessità di vivere la fede cristiana nell'assoluta povertà, ma da cui si distanzierà per l'atteggiamento assunto nei confronti della Chiesa. Infatti, mentre Francesco si sottomise all'autorità ecclesiastica, Pietro Valdo se ne allontanò, "ponendo il testo evangelico

predestinazione è divenuta dominante, «la Chiesa è concepita e definita, con un semplicismo sconcertante, come la comunità dei predestinati. Questa esiste ovunque Dio ha dei veri adoratori; essa è dunque, come tale, indiscernibile e invisibile; ma né Hus né Wicliff parlano espressamente di "Chiesa invisibile"». Inoltre, conclude l'A., "si può rimproverare a una tale "ecclesiologia" di considerare le cose solamente dal punto di vista dell'individuo, di trasporre sulla Chiesa stessa delle affermazioni concernenti gli individui. Se vi sono dei giusti e dei peccatori, dei veri cristiani e dei cristiani solo in apparenza, non vi sono per ciò stesso due Chiese». Per una corretta ricostruzione del pensiero ecclesiologico di Agostino si veda V. GROSSI, La Chiesa di Agostino. Modelli e simboli, EDB, Bologna, 2012.

${ }^{81}$ Cfr. G. TOURN, I valdesi identità e storia, Claudiana, Torino, 2003, p. 31.

82 Per esso si intende il sermone rivolto da Cristo ai suoi discepoli su una montagna al nord del mare di Galilea, vicino Cafarnao, riportato nel Vangelo secondo Matteo 5,1-7,29. Cfr. J. RATZINGER, Gesù di Nazaret, Rizzoli, Milano, 2007, pp. 93-125.

83 "Beati i poveri in spirito, perché di essi è il regno dei cieli" $(\mathrm{Mt}, 5,7)$.

${ }^{84}$ Cfr. G. TOURN, I valdesi identità e storia, cit., p. 32. In maniera più ampia ricorda G.G. MERLO, Pauperes spiritu valdesi, in Bollettino della Società di Studi Valdesi, 2016/219, p. 21, "Pauperes esse decrevimus" significava diventare "pauperes spiritu" e, quindi fare proprie le beatitudini del "discorso della montagna" di Matteo 5,3-10; fare proprie tutte le beatitudini così dei poveri in spirito, come degli afflitti, dei miti, degli affamati e assetati di giustizia, dei misericordiosi, dei puri di cuore, dei facitori di pace, dei perseguitati. Siamo così al centro identitario di un'esperienza religiosa che, partendo dalla professione di povertà, se ne va al di là, per arrivare a una totale adesione al vangelo: "vocant se Pauperes spiritu"». 
come principio di autorità" 85 . La ragione del conflitto era rappresentata dalla presunzione, presente sin dall'origine nella laica societas valdese, di poter predicare liberamente l'Evangelo usurpando, cosi, l'officium praedicationis riservato al genus clericorum ${ }^{86}$. La radicalizzazione della vicenda portò dapprima l'Arcivescovo di Lione, Jean Bellesmains, ad allontanare Pietro Valdo e i suoi seguaci dalla Francia sud-orientale nel $1183^{87}$, e 1'anno seguente, Papa Lucio III, in occasione del Concilio di Verona, a scomunicarli con la decretale Ad abolendam utilizzando l'espressione significativa "eos qui se Humiliatos vel Pauperes de Lugduno falso nomine mentiuntur" 88 .

Dopo secoli di clandestinità, trascorsi nelle aree alpine del Piemonte, il movimento valdese riprese la propria espansione con la Riforma protestante ${ }^{89}$, di cui accolse le principali dottrine con il Sinodo di Chanforan del 12 settembre $1532^{90}$. Questa scelta, sintesi delle eterogenee correnti del valdismo, aveva garantito loro una piena autonomia confessionale, anche se, venendo tale comunità assorbita nel mare magnum protestante, era andata dispersa l'originalità che da sempre l'aveva contraddistinta ${ }^{91}$. Pertanto, anche i valdesi-riformati patirono la controffensiva cattolica del XVI secolo, che si articolò nella repressione attuata dai nuovi Ordini religiosi (cappuccini e gesuiti), dall'Inquisizione e dall'azione dei Principi cattolici Filippo II di Spagna, Enrico II di Francia, Maria Tudor d'Inghilterra ed Emanuele Filiberto di Savoia ${ }^{92}$.

Si determinò, così, una nuova 'ghettizzazione valdese' da cui la

${ }^{85}$ Cfr. G. TOURN, I Valdesi. La singolare vicenda di un popolo-chiesa, $4^{\text {a }}$ ed., Claudiana, 2008, p. 43.

${ }^{86}$ Cfr. G.G. MERLO, Pauperes spiritu, cit., p. 17.

${ }^{87}$ Cfr. G. TOURN, I Valdesi. La singolare vicenda, cit., p. 13.

${ }^{88}$ Cfr. G.G. MERLO, Pauperes spiritu, cit., p. 18.

89 Sulla diffusione della Riforma protestante in Italia analitica è la ricostruzione operata da S. CAPONETTO, La Riforma protestante nell'Italia del Cinquecento, 2a ed., Claudiana, Torino, 1997.

90 Cfr. A.A. HUGON, Storia dei Valdesi. Dal sinodo di Chanforan all'Emancipazione, Claudiana, Torino, 1989, vol. II, pp. 13-18.

${ }^{91}$ Cfr. G. TOURN, I Valdesi identità e storia, cit., p. 42, secondo il quale «i discepoli di Valdo, i "Poveri di Lione" che avevano vissuto come un movimento di rinnovamento della chiesa romana sono una cosa, ma una volta entrati nel mondo protestante sono diventati altra cosa, hanno cambiato tutto: le idee, le abitudini, la teologia. Ormai sono protestanti e basta, e non si possono più attribuire il nome valdese».

${ }_{92}$ Cfr. G. TOURN, I Valdesi identità e storia, cit., p. 30 e p. 43. 
comunità de qua si emanciperà 93 , definitivamente, solo il 17 febbraio 1848 allorquando Carlo Alberto di Savoia, Principe sovrano, sottoscritte le Lettere Patenti, concesse alle popolazioni valdesi, e poi alla minoranza ebraica, i diritti civili e politici ${ }^{94}$. In questo modo, i valdesi, nel più generale clima risorgimentale ${ }^{95}$, riprenderanno la loro diffusione nell'Italia peninsulare, "con lo stesso spirito del XII e del XVI secolo, e con lo stesso intendimento: partecipare al rinnovamento della coscienza religiosa e civile dei propri concittadini" 96.

\section{9 - Il movimento dei Lollardi}

Gli ultimi anni del XIV secolo costituiscono il periodo nel quale maggiore è stata la repressione, ex parte catholica, dei movimenti del dissenso ${ }^{97}$. Questo è, però, anche il tempo in cui apparve in Inghilterra il francescano John Wycliff, comunemente, quanto impropriamente, definito il vero precursore della Riforma protestante ${ }^{98}$, la cui vicenda personale e le cui teorie saranno di ispirazione per la "rivoluzione hussita"99.

Il doctor evangelicus ${ }^{100}$, teologo e professore universitario a Oxford, era titolare della ricca Parrocchia regia di Lutterworth, ma ciò non gli impedì di formulare critiche serrate contro la Chiesa, di cui denunciava le enormi ricchezze, finanziarie e terriere ${ }^{101}$. Egli, negando la tradizione cristiana, considerava come unico fondamento della fede la Bibbia (De veritate scripturae 1379), di cui promosse una traduzione in lingua

93 Cfr. A.A. HUGON, Storia dei Valdesi, cit., pp. 297-302.

94 Come ricorda, infatti, G. TOURN, I Valdesi. La singolare vicenda, cit., p. 218, «per quanto concerne la libertà religiosa la situazione rimaneva immutata e le Patenti lo precisavano: "nulla però è rinnovato quanto all'esercizio del loro culto [...]". Libertà civile dunque, non libertà di culto. La religione cattolico-romana restava la religione dello Stato».

95 Sul tema diffusamente G. TOURN, Risorgimento e chiese cristiane, Claudiana, Torino, 2011.

96 Cfr. G. TOURN, I valdesi identità e storia, cit., p. 30.

${ }^{97}$ Cfr. G. TOURN, I valdesi identità e storia, cit., p. 29.

98 Cfr. K. BILMEYER, H. TUECHLE, L'epoca delle riforme, cit., p. 116.

${ }^{99}$ Cfr. G. TOURN, I Valdesi. La singolare vicenda, cit., p. 79.

100 Così veniva definito John Wycliff dai suoi seguaci, avendo egli, con molta probabilità, personalmente tradotto il Nuovo Testamento. Cfr. G. TOURN, I Valdesi. La singolare vicenda, cit., p. 79.

${ }^{101}$ Cfr. W.G. NAPHY, La rivoluzione protestante, cit., p. 5. 
inglese $^{102}$, alla quale ogni vero cristiano aveva il diritto di accedere in modo diretto e individuale. Per questo motivo Wycliff negava ogni autorità, spirituale e temporale, alla gerarchia ecclesiastica, identificata con la "Chiesa visibile", alla quale, nel De Ecclesia (1377-78), uno dei suoi scritti polemici, opponeva, sullo stile donatista del V secolo103, la "Chiesa invisibile" dei predestinati104, la Congregatio praedestinatorum o Communio electorum ${ }^{105}$.

In particolare, il pensiero wycleffita rigettava il potere di scomunica del Pontefice e dei Vescovi (De potestate Papae 1378), la dottrina della transustanziazione ${ }^{106}$ (De Eucharistia 1379), ma anche il valore della confessione auricolare, il celibato ecclesiastico e tutta una serie di pratiche devozionali, dalle indulgenze alle messe per i defunti ${ }^{107}$. Inoltre la Chiesa era considerata priva del potere temporale e, per questo, sottomessa all'autorità civile, alla quale dovevano essere cedute tutte le ricchezze accumulate dal clero nel corso dei secoli ${ }^{108}$.

In maniera del tutto singolare le ridette teorie, almeno finché il suo autore $\mathrm{fu}$ in vita, non subirono alcuna condanna, nonostante i processi giudiziari che la Chiesa ufficiale inglese aveva celebrato a suo carico. Si ricorda, a tal proposito, un'istruttoria svolta contro Wycliff dall'allora Arcivescovo di Canterbury, Simon Sudbury, conclusasi, nel marzo 1378, con una semplice ammonizione ${ }^{109}$. Le ragioni di questo atteggiamento

102 Cfr. I.C. LEVY, John Wyclif: Scriptural Logic, Real Presence, and the Parameters of the Orthodoxy, Marquette University Press, Milwaukee, 2003.

103 Il donatismo, che prende il nome da Donato di Cartagine, è un movimento scismatico della Chiesa africana, durato dalla fine della persecuzione di Diocleziano all'invasione musulmana. In particolare sulla ecclesiologia donatista interessante è il contributo di A. ROSSI, Muscae Moriturae Donatiste Circumvolant. La costruzione di identità "plurali" nel cristianesimo dell'Africa romana, Ledizioni, Milano, 2013, pp. 183-248.

104 Diffusamente sul tema M. FuMAGALLI BEONIO BROCCHIERI (a cura di), La chiesa invisibile. Riforme politico-religiose nel basso Medioevo, Feltrinelli, Milano, 1979.

105 Cfr. Y. CONGAR, Vera e falsa riforma, cit., p. 282.

106 Per essa si intende la trasformazione nel rito della messa del pane e del vino nel vero corpo e nel vero sangue di Cristo, surrogandola con la teoria della remanenza, per la quale anche dopo la consacrazione rimanevano la sostanza del pane e del vino. Cfr. AA. VV., Declino dell'impero e del papato e sviluppo degli stati nazionali, a cura di Z.N. BROOKE, C.W. Previté-Orton, J.R. TANNER in Storia del Mondo Medievale, Aldo Garzanti, Milano, 1980, vol. VI, p. 803.

107 Cfr. K. BILMEYER, H. TUECHLE, L'epoca delle riforme, cit., p. 118.

108 Cfr. F. BUZZI, Breve storia del pensiero protestante da Lutero a Pannenberg, Ancora, Milano, 2007, p. 8.

${ }^{109}$ Cfr. K. BILMEYER, H. TUECHLE, L'epoca delle riforme, cit., p. 118. 
vanno storicamente ravvisate nella protezione che i Regnanti inglesi assicurarono a John Wycliff e ai suoi seguaci ${ }^{110}$, i cosiddetti 'lollardi'111, che si distinsero per una vera e propria azione missionaria sul modello evangelico della Chiesa primitiva.

Bisogna attendere Enrico IV per registrare un significativo cambio di rotta: infatti il Monarca Lancaster fece approvare dal Parlamento inglese, nel 1400, il De haeretico comburendo, con cui si introdusse oltre Manica l'Inquisizione e vennero mandati al rogo i lollardi pertinaci. Quanto a John Wycliff, il Concilio di Costanza (1415) dichiarò eretiche le sue proposizioni ${ }^{112}$, accanendosi contro i di lui resti mortali che vennero disseppelliti e bruciati ${ }^{113}$.

\section{0 - La rivoluzione boema}

Nonostante ciò, la diffusione centroeuropea del pensiero wycleffita fu

110 Cfr. F. BUZZI, Breve storia, cit., p. 7.

111 Sul significato del termine e sulla diffusione del movimento la dottrina è alquanto divisa: secondo W.G. NAPHY, La rivoluzione protestante, cit., p. 5, l'espressione lollardi "probabilmente deriva dall'olandese lollaerd, ovvero che si perde in ciance", il quale ritiene inoltre che "il movimento, a conti fatti, ottenne poco successo e in breve tempo finì nel dimenticatoio". Diversa è la ricostruzione di P.G. WALLACE, La lunga età, cit., p. 85, secondo cui lollardo inizialmente significava "che prega a bassa voce" ma poi "passò ad indicare genericamente qualsiasi persona accusata di eresia", mentre "il movimento organizzato cessò di esistere dopo il 1410". Infine nella elaborazione di K. BILMEYER, H. TUECHLE, L'epoca delle riforme, cit., p. 119, “i missionari ambulanti furono dai cattolici chiamati lollardi, cioè seminatori di zizzania (da lollium)". Per un ulteriore approfondimento sulla materia si rimanda a M. ASTON, Lollards and Reformers. Images and Literacy in Late Medieval Religion, The Hambledon Press, London, 1984.

112 In realtà, già in occasione del Sinodo di Londra del 17 maggio 1382, detto del terremoto, convocato dall'allora Arcivescovo di Canterbury, Guglielmo di Courtenay, ben 24 proposizioni di Wycliff erano state condannate: 10 come eretiche e 14 come erronee. Cfr. Z.N. BROOKE, C.W. PREVITÉ-ORTON, J.R. TANNER, Declino dell'impero e del papato, cit., p. 793.

113 Infatti, "morto in comunione con la Chiesa, Wycliffe venne seppellito a Lutterworth, ma trent'anni più tardi, quando in Boemia si verificarono tutte le conseguenze del suo insegnamento, il concilio di Costanza lo condannò e ordinò che le sue ossa fossero rimosse dalla terra consacrata. L'ex lollardo, Repingdon, allora vescovo di Lincoln, cui venne affidato questo compito, non obbedì; ma Fleming, che pure in gioventù era stato coinvolto nell'eresia, incalzato dalla perentoria richiesta di Martino V, eseguì l'ordine; così nel 1428 le ossa di Wycliffe vennero dissotterrate, bruciate, e le ceneri disperse nello Swift". Cfr. Z.N. BROOKE, C.W. PREVITÉ-ORTON, J.R. TANNER, Declino dell'impero e del papato, cit., p. 795. 
considerevole ${ }^{114}$. In particolare, gli scritti del lollardo giunsero per via accademica in Boemia, territorio nel quale alla fine del XIV secolo erano ancora presenti residui ereticali catari ${ }^{115}$ e valdesi, attesa la relazione tra l'Università di Oxford e quella di Praga, rinsaldata dall'unione dinastica tra la Corona inglese e quella ceca116. In questo contesto, fu essenziale il contributo di Jan Hus, docente di teologia e rettore dell'Università Carolina ${ }^{117}$, il quale era venuto in contatto con le opere di John Wycliff intorno al 1398, grazie al suo compagno di studi Girolamo di Praga, subendone fortemente l'influsso ${ }^{118}$.

La circolazione delle dottrine del riformatore inglese generò nell'Università di Praga un forte dibattito, che portò il corpo docente, a prevalenza tedesca in virtù della colonizzazione teutonica della Boemia, iniziata nell'alto medioevo, dapprima a censurare le proposizioni lollarde (1403), e poi, vista la protezione offerta dal Re Venceslao alla figura di Hus, ad abbandonare la città, il 16 maggio 1409, unitamente a un gruppo di studenti, circa un migliaio, per fondare l'Università di Lipsia119.

Nello stesso tempo, Jan Hus, dal pulpito della Cappella di Betlemme in Praga, iniziò una campagna di evangelizzazione in lingua ceca $^{120}$ : criticando le eccessive ricchezze della Chiesa ${ }^{121}$, affermando la

114 Cfr. H. KAMINSKY, A History of the Hussite Revolution, University of California Press, Berkeley, 1967, pp. 23-55.

115 I catari (o albigesi) sono eretici dualisti medievali diffusi nella Francia settentrionale e meridionale del XIII secolo. Essi, dotati di una propria gerarchia, professavano, in polemica con la Chiesa, un rinnovamento morale fondato sull'opposizione tra bene e male, spirito e materia. Sulle differenze tra catari e valdesi si veda G. TOURN, I valdesi identità e storia, cit., pp. 32-33.

116 A tal proposito, nel 1382, venne celebrato il matrimonio tra il Re d'Inghilterra Riccardo II con Anna di Lussemburgo, figlia dell'Imperatore Carlo IV, sorella di Venceslao Re di Boemia. Cfr. F. BUZZI, Breve storia, cit., p. 7.

117 Infatti l'Università di Praga venne fondata da Carlo IV di Lussemburgo il 7 aprile 1348 e, in ragione di ciò, è anche nota come Università Carlo IV.

118 Cfr. A. QUERENGÄSSER, S. LUNYAKOV, Le guerre Hussite. Gli eserciti e gli armamenti, le tattiche le battaglie e le campagne 1419-1437, Soldiershop Publishing, Zanica (Bergamo), 2020, vol. I, p. 10.

${ }^{119}$ Cfr. K. BILMEYER, H. TUECHLE, L'epoca delle riforme, cit., p. 121.

120 Infatti ai ministri della Cappella di Betlemme, fondata a Praga nel 1391, era stato imposto l'obbligo di predicare due volte ogni domenica o giorno festivo in lingua ceca. Cfr. AA. VV., L'autunno del medioevo e la nascita del mondo moderno, a cura di Z.N. BROOKE, C.W. Previté-Orton, J.R. TANNER in Storia del Mondo Medievale, Garzanti, Milano, 1981, vol. VII, p. 118.

${ }^{121}$ Cfr. W.G. NAPHY, La rivoluzione protestante, cit., p. 5. 
superiorità della Scrittura e del potere civile su quello ecclesiastico ${ }^{122}$, e richiedendo anche per i laici la Comunione sotto le due specie, del pane e del vino (utraquismo), come conseguenza della loro equiparazione con il clero $^{123}$.

Questo tipo di predicazione assicurò al riformatore hussita, nel 1412, la scomunica maggiore del Papa pisano124 Giovanni XXIII e l'interdetto sul luogo ove egli soggiornava, e quindi il bando del Re Venceslao, che lo obbligò ad abbandonare Praga, costringendolo all'esilio' $^{125}$. In questa circostanza, Hus scrisse il suo De Ecclesia (1413), opera di chiara ispirazione wycleffita, nel quale la vera Chiesa di Cristo non coincide con quella romana, la Congregatio fidelium fondata sulla partecipazione ai sacramenti e sulla gerarchia, ma si identifica con la Comunità dei predestinati, la Universitas praedestinatorum che rappresenta il Corpo mistico di Cristo ${ }^{126}$.

"Quindi, né il Papa è il Capo né i Cardinali sono tutto il Corpo della santa Chiesa universale, ma solo Cristo è il Capo di quella Chiesa, e i singoli predestinati ne sono insieme e il Corpo e un membro, perché una sola è la persona, la stessa Sposa di Cristo Gesù"127.

In questo modo, Hus contesta anche l'autorità del Pontefice sulla Chiesa: infatti nel capitolo del De Ecclesia dedicato al primato di Pietro, egli, seguendo Sant'Agostino, identifica il termine 'pietra', utilizzato dall'evangelista Matteo ${ }^{128}$, non con Pietro ma con Cristo medesimo. "Questo dice Agostino, insegnando, d'accordo con 1'Apostolo, che solo Cristo è il fondamento e la pietra su cui, cioè su Cristo, è fondata la

122 Cfr. L. FELICI, La Riforma protestante, cit., p. 33.

123 Infatti, secondo A. QUERENGÄSSER, S. LUNYAKOV, Le guerre Hussite, cit., vol. I, p. 10, «a quel tempo, era consuetudine che solo i sacerdoti bevessero vino alla Comunione, mentre il resto della congregazione riceveva solamente l'ostia consacrata. Hus pretese - poco prima della sua morte - che tutti ricevessero una parte del sangue di Cristo. Poiché questo veniva consegnato in un calice, la gente cominciò a chiamare i suoi seguaci come "Calixtiner" (dal latino "calix" per "calice") o anche "Utraquists" (dal latino "sub utraque parte" = in entrambi i tipi)».

124 Come ricorda C. FANTAPPIÈ, Storia del diritto canonico, cit., p. 159, questo appellativo gli era stato attribuito "perché eletto dal concilio di Pisa del 1409".

125 Cfr. P.G. WALLACE, La lunga età della Riforma, cit., p. 88.

${ }^{126}$ Cfr. G. MARTINA, Storia della Chiesa, cit., p. 109.

${ }^{127}$ Cfr. J. HUS, Il primato di Pietro. Dal De ecclesia, 2a ed., Claudiana, Torino, 2009, p. 45.

128 "Tu sei Pietro e su questa pietra edificherò la mia Chiesa e le porte degli inferi non prevarranno contro di essa" (Mt, 16,18). 
Chiesa"129.

La repressione delle eresie di John Wycliff e Jan Hus, ancora persistenti, considerata la lacerante situazione vissuta dalla Chiesa cattolica del tempo, divisa come noto nelle tre obbedienze pontificie ${ }^{130}$, si raggiunse con la celebrazione del Concilio di Costanza, indetto nel novembre 1414 da Giovanni XXIII, su sollecitazione dell'Imperatore Sigismondo ${ }^{131}$.

La causa fidei fu affrontata e risolta nella VIII sessione generale del 4 maggio 1415, con la sanzione delle tradizionali proposizioni di Wycliff, e nella XV sessione generale del 6 luglio 1415, con la condanna a eretico pertinace di Hus che, nonostante il salvacondotto imperiale, fu consegnato al braccio secolare che lo mise al rogo. Medesimo destino raggiunse un anno dopo Girolamo di Praga. Il Concilio vietò, inoltre, l'uso del calice ai laici ${ }^{132}$

\section{1 - Le guerre hussite}

L'uccisione di Jan Hus non coincise con la scomparsa del movimento riformatore boemo che, nell'ottobre 1419, formalizzò le proprie richieste elaborando gli 'articoli di Praga': libertà di predicazione, concessione del calice ai laici, abolizione del potere temporale e della proprietà del clero, punizione dei peccati mortali e di tutte le deviazioni contrarie alla legge divina ${ }^{133}$.

Ben presto, però, la rivolta religiosa e quella politica si saldarono ${ }^{134}$. Infatti, un gruppo di ribelli si rese autore della "defenestrazione di Praga"135, determinando la reazione dell'Imperatore Sigismondo, nuovo

129 Cfr. J. HUS, Il primato di Pietro, cit., p. 59.

130 Infatti, nel 1414, la Chiesa cattolica era segnata dalla presenza di tre Pontefici regnanti: Gregorio XII a Roma, Benedetto XIII ad Avignone, e Giovanni XXIIII a Pisa. Cfr. Z.N. Brooke, C.W. Previté-Orton, J.R. TANNER (a cura di), L'autunno del medioevo, cit., pp. 5-7.

${ }^{131}$ Cfr. N.P. TANNER, I Concili, cit., p. 73.

${ }^{132}$ Cfr. K. BILMEYER, H. TUECHLE, L'epoca delle riforme, cit., p. 122.

133 Cfr. Z.N. BrooKe, C.W. Previté-Orton, J.R. TANner (a cura di), L'autunno del medioevo, cit., p. 155.

${ }^{134}$ Cfr. T.A. FUDGE, Jan Hus. Religious Reform and Social Revolution in Bohemia, I.B. Tuaris, London-New York, 2010.

135 A tal proposito, A. QUERENGÄSSER, S. LUNYAKOV, Le guerre Hussite, cit., vol. I, p. 13, ricordano che "il 30 luglio 1419 un gruppo infuriato di cittadini praghesi prese 
Re di Boemia, che inviò il proprio esercito per reprimere i tumulti, sollecitando Martino V, il nuovo e unico Pontefice ${ }^{136}$, a intraprendere contro di loro una crociata ${ }^{137}$. La resistenza venne brillantemente affidata all'ala radicale dei 'taboriti', cosiddetti dalla loro roccaforte sul monte Tabor, che, guidati da Jan Žižka, divennero i veri protagonisti delle sanguinose 'guerre hussite'(1420-1431)138.

L'incapacità di risolvere in modo cruento la vicenda impose il ricorso alla diplomazia. I riformatori boemi furono convocati al Concilio di Basilea, radunato da Martino V per il 1431, durante il quale venne loro offerta una conciliazione basata sui vecchi articoli di Praga. L'ala moderata del movimento, gli utraquisti o callistini, accolse di buon grado la proposta, raggiungendo un accomodamento nelle 'Compattate di Praga' del 30 novembre 1433139 , mentre i taboriti, che avanzavano propositi meno concilianti, furono annientati, insieme alle loro richieste ${ }^{140}$, al culmine della guerra civile intrapresa conto gli stessi utraquisti, congiunti ai cattolici, nel maggio 1434 a Lipany ${ }^{141}$.

Di seguito, i taboriti superstiti diedero vita, con il contributo di un

d'assalto il Municipio della Città Nuova per liberare i compagni di fede che vi si trovavano imprigionati. Così facendo, si imbatterono nel podestà, il suo vice, due consiglieri, cinque anziani della comunità e uno scudiero: tutti quanti vennero gettati fuori dalla finestra".

136 Per K. BILMEYER, H. TUECHLE, L'epoca delle riforme, cit., p. 75, "nel conclave, tenutosi a Costanza nella loggia dei mercanti, l'11 novembre 1417 la totalità dei voti si concentrò sul cardinale Odo Colonna = Martino V (1417-31). In lui la Chiesa ricevette il nuovo capo universalmente riconosciuto e degno, nelle cui mani da quel momento venne a trovarsi la direzione del concilio".

${ }^{137}$ Cfr. A. QUERENGÄSSER, S. LUNYAKOV, Le guerre Hussite, cit., vol. II, p. 5.

${ }^{138}$ Cfr. N.P. TANNER, I Concili, cit., p. 79.

139 Cfr. Z.N. Brooke, C.W. Previté-Orton, J.R. TANNER (a cura di), L'autunno del medioevo, cit., pp. 160-161.

140 Ricostruisce fedelmente le richieste taborite W.G. NAPHY, La rivoluzione protestante, cit., pp. 6-7, secondo il quale «l'ala più radicale degli hussiti [...] rifiutava il dogma della transustanziazione $[\ldots]$ negava altresì ogni definizione del sacerdozio come separato dal resto della comunità dei credenti $[. .$.$] sosteneva che il Cristianesimo della prima Chiesa \mathrm{e}$ il Nuovo Testamento chiamassero a una separazione radicale dalla società in generale $\mathrm{e}$, in particolare, dallo Stato. Enfatizzava inoltre, l'esortazione di Cristo a "porgere l'altra guancia". Si opponeva quindi a ogni forma di violenza, punizione capitale, servizio militare e giuramento (anche in tribunale). Molti di questi temi ricompariranno nella storia successiva della Riforma». Conformemente L. FELICI, La Riforma protestante, cit., p. 33.

${ }^{141}$ Cfr. A. QUERENGÄSSER, S. LUNYAKOV, Le guerre Hussite, cit., vol. II, pp. 80-85. 
Vescovo valdese ${ }^{142}$, alla setta religiosa della 'Unità dei Fratelli della legge di Cristo' (Unitas Fratrum), mentre gli utraquisti videro confermate le Compattate di Praga nell'Intesa di Iglau, siglata con la Chiesa romana nel $1436^{143}$.

L'istituzionalizzazione del movimento ussita in Chiesa nazionale boema ${ }^{144}$ ha rappresentato, senza dubbio, la prima e permanente frattura nella Respublica Christiana ${ }^{145}$. Nacque, infatti, dall'azione riformatrice di Jan Hus e dal contributo turbolento dei suoi proseliti, una vera e propria confessione, nel senso moderno del termine assai simile, nell'impostazione gerarchica, nel culto e nella teologia, a quella di Roma; contraddistinta, però, dal rifiuto della supremazia papale ${ }^{146}$, che rappresenterà per la Chiesa d'Inghilterra il 'sommo analogato' 147.

142 Per confermare lo stabile rapporto valdo-hussita, G. TOURN, I valdesi identità $e$ storia, cit., p. 81, ricorda che "dal movimento hussita i valdesi ricevettero una cultura teologica. I teologi di Praga, impegnati in una riflessione a carattere biblico, avevano spesso proseguito la ricerca valdese, portandola a livello universitario. [...] Importante fu però anche l'influenza hussita sul terreno pratico. Un nuovo slancio missionario proveniente dalla Boemia rianima la diaspora valdese; i ministri tedeschi soggiornavano presso le comunità taborite, mantenevano contatti e alcuni di questi uomini [...] sognano un grande movimento evangelico che partendo dalla Boemia, si irradi attraverso l'Impero lungo le vie della diffusione valdese".

143 Cfr. H.G. BECK, K.A. FINK, J. GLAZIK, E. ISERLOH, Tra Medioevo e Rinascimento. Avignone - Conciliarismo - Tentativi di riforma (XIV-XVI sec.), in. H. JEDIN (diretta da), Storia della Chiesa, Cooperativa Edizioni Jaca Book, Milano, 1993, 2a ed., vol. II, p. 228.

${ }^{144}$ Cfr. É.G. LÉONARD, La Riforma, cit., p. 28.

145 Cfr. P.G. WALLACE, La lunga età della Riforma, cit., p. 90.

146 Cfr. W.G. NAPHY, La rivoluzione protestante, cit., p. 7.

147 Infatti la Chiesa d'Inghilterra, uniformandosi al principio del "cuius regio, eius et religio", ha riprodotto i caratteri tipici della Chiesa nazionale, in cui l'unica religione consentita è quella professata dal Sovrano. A tal proposito si consenta il rinvio a $\mathbf{R}$. GRANATA, Riforma protestante ed ecclesiologia cristiana: il modello di "governo» della Chiesa anglicana, in AA. VV., Il diritto come "scienza di mezzo". Studi in onore di Mario Tedeschi, a cura di M. D’ARIENZO, Luigi Pellegrini, Cosenza, 2017, vol. II, pp. 1188-1189. 